\title{
Linear and Nonlinear Dust Ion Acoustic Waves in a Dense Quantum Magneto Plasma
}

\author{
Elham Emadi and Hossein Zahed* \\ Physics Department, Sahand University of Technology, Tabriz, Iran \\ *Corresponding Author Email: zahed@ sut.ac.ir \\ Regular paper: Received: Oct. 15, 2018, Revised: Apr. 6, 2019, Accepted: May. 31, 2019, \\ Available Online: June. 30, 2020, DOI: 10.29252/ijop.14.1.39
}

\begin{abstract}
In this article, linear and nonlinear dust ion acoustic (DIA) waves are studied in a magnetized quantum dusty plasma which consists of inertialess electrons and positrons, cold ions and negatively charged dust grains. For this purpose, quantum Hydrodynamic model (QHD) and reductive perturbation method are employed. To investigate linear and nonlinear waves, dispersion relation and a quantum ZakharovKuznetsov (ZK) equation are derived respectively. A stationary solution of the ZK equation is obtained to investigate the effects of plasma parameters on the amplitude of the solitons.
\end{abstract}

KEYWORDS: Dust ion acoustic waves, quantum Hydrodynamic model, quantum magnetoplasma.

\section{I.INTRODUCTION}

Ordinary plasma is composed of freely moving charged particles which are particularly electrons and ions. This could be implied that few electron volts $\left(10^{5} \mathrm{~K}\right)$, break neutral atoms and molecules to free electrons and ions. At high temperatures and low densities, thermal energy dominates, electrons and ions interact under classical dynamical laws subject to Newtonian mechanics and Maxwellian statistical physics. Quantum plasmas are known to exist under high-density conditions where the plasma electrons behave fully quantum mechanically such as plasmas which are created in many modern experiments with short laser pulses, ion beams or combination of both $[1,2]$. Quantum effects also are expected to occur in many dense astrophysical plasmas
[3], for example in the core of the giant planets such as Jupiter, Saturn, and brown or white dwarf stars [4], Neptune [5-8], neutron stars [9]. Furthermore the exotic state of the Universe after the Big Bang is believed to have been a quantum plasma. In all these cases, a description based on classical mechanics and thermodynamics, or classical kinetic theory is unsuccessful. A theoretical description based on the Schrodinger equation and quantum many-body theory involving methods such as quantum statistics, quantum kinetic theory, Hartree-Fock and nonequilibrium Green's functions (NEGF) use to carry out such investigations. Quantum hydrodynamic (QHD) model is a popular model which widely is used for description of hydrodynamic and statistical behaviors of plasma particles in quantum ranges[10, 11]. This model is a generalization of the classical fluid model of plasmas where QHD transport equations are expressed in terms of conservation laws for particle momentum and energy. Zakharov-Kuznetsov (ZK) has been extensively used to study the IA waves in magnetized plasmas[12, 13]. ZK equation governs the behavior of weakly nonlinear waves in a plasma consisting of cold ions and hot isothermal electrons in the presence of a uniform magnetic field and it has been almost employed for other conditions. Moslem et al. derived the $\mathrm{ZK}$ equation for electron-ion quantum plasma[14]. The authors have also examined the stability of the wave and discussed the importance of their results. Khan and et al. studied DIA solitary waves in a magnetized quantum dusty plasma by using the quantum hydrodynamic model and derived 
ZK equation [15]. In the present paper, we study the propagation of linear and nonlinear acoustic DIA waves in a dense magnetized quantum plasma consisting of inertialess electrons and positrons, cold ions and negatively charged dust grains by employing the QHD model. We derive dispersion relation and $\mathrm{ZK}$ equation taking into account the effects of quantum pressure and Fermi-Dirac statistics for the lighter species electrons and positrons. The manuscript is organized as follows. The governing equations are given in Sec II. Dispersion relation is derived for linear waves in section III and The ZK equation is derived for nonlinear waves in Sec IV. Section $\mathrm{V}$ is devoted to the solitary wave solution, results and discussions and The conclusion is given in section VI.

\section{GOVERNING EQUATIONS}

We study the linear and nonlinear propagation of DIA waves in a dense quantum magneto plasma composed of inertialess electrons and positrons, cold ions and negatively charged dust grains immersed in a uniform external magnetic field acting along the $\mathrm{z}$-direction $\left(\mathbf{B}_{\mathbf{0}}=\hat{\mathbf{k}} B_{0}\right)$. At equilibrium the charge neutrality requires $1+p=e+d$, where $p=\frac{n_{p 0}}{n_{i 0}}$, $e=\frac{n_{e 0}}{n_{i 0}}$, and $d=\frac{Z_{d 0} n_{d 0}}{n_{i 0}}, n_{p 0}, n_{e 0}, n_{i 0}$, and $n_{d 0}$ are respectively the equilibrium number density of positron, electron, ion, and dust. $Z_{d 0}$ is the dust charging state. The nonlinear dynamics of DIA wave in this plasma model, is governed by the following QHD equations

$$
\begin{gathered}
\frac{\partial n_{d}}{\partial t}+\vec{\nabla} \cdot\left(n_{d} \mathbf{u}_{\mathbf{d}}\right)=0 \\
\frac{\partial n_{i}}{\partial t}+\vec{\nabla} \cdot\left(n_{i} \mathbf{u}_{\mathbf{i}}\right)=0 \\
\nabla^{2} \varphi=\mu_{d}(1-d) n_{e}+d n_{d}-n_{i}+ \\
\left(1-\mu_{d}\right)(1-d) n_{p}
\end{gathered}
$$

$$
\begin{aligned}
& \frac{\partial \mathbf{u}_{\mathbf{d}}}{\partial t}+\left(\mathbf{u}_{\mathbf{d}} \cdot \vec{\nabla}\right) \mathbf{u}_{\mathbf{d}}=\mu \vec{\nabla} \phi-\mu \Omega_{c i}\left(\mathbf{u}_{\mathbf{d}} \times \hat{\mathbf{z}}\right) \\
& \frac{\partial \mathbf{u}_{\mathbf{i}}}{\partial t}+\left(\mathbf{u}_{\mathbf{i}} \cdot \vec{\nabla}\right) \mathbf{u}_{\mathbf{i}}=-\vec{\nabla} \phi+\Omega_{c i}\left(\mathbf{u}_{\mathbf{i}} \times \hat{\mathbf{z}}\right)-\sigma n_{i} \vec{\nabla} n_{i} \\
& \vec{\nabla} \phi=\Omega_{c i}\left(\mathbf{u}_{\mathbf{e}} \times \hat{\mathbf{z}}\right)+n_{e} \vec{\nabla} n_{e}-\frac{H^{2}}{2} \vec{\nabla}\left(\frac{\nabla^{2} \sqrt{n_{e}}}{\sqrt{n_{e}}}\right) \\
& \vec{\nabla} \phi=\Omega_{c i}\left(\mathbf{u}_{\mathbf{p}} \times \hat{\mathbf{z}}\right)-n_{p} \vec{\nabla} n_{p}-\frac{H^{2}}{2} \vec{\nabla}\left(\frac{\nabla^{2} \sqrt{n_{p}}}{\sqrt{n_{p}}}\right)
\end{aligned}
$$

The number density $n_{j}$, is normalized by its unperturbed density $n_{j 0}$, where $j=e$ for electron, $i$ for ion, $p$ for positron and $d$ for dust particle. The electrostatic wave potential $\varphi$ is normalized by $\frac{2 k_{B} T_{F e}}{e}$. The fluid velocity $u_{j}(j=e, i, p, d)$ is normalized by the quantum ion-acoustic speed $C_{s i}$. The space and time coordinates $x$ and $t$ are normalized respectively, by the quantum Debye length $\lambda_{D}$ and the ion plasma frequency $\omega_{p i}$. Further, $\lambda_{D}=\sqrt{\frac{2 k_{B} T_{F e}}{4 \pi e^{2} n_{i 0}}}, \omega_{p i}=\sqrt{\frac{4 \pi e^{2} n_{i 0}}{m_{i}}}, C_{s i}=\sqrt{\frac{2 k_{B} T_{F e}}{m_{i}}}$
$, \mu=\frac{Z_{d 0} m_{i}}{m_{d}}, \quad \mu_{d}=1+\frac{P}{1-d}, \gamma=\frac{T_{F i}}{T_{F p}}, \sigma=\frac{T_{F i}}{T_{F e}}$,
$\Omega_{c i}=\frac{\omega_{c i}}{\omega_{p i}}$, where $T_{F j}(\mathrm{j}=e, i, p)$ is Fermi temperature and $\omega_{c i}$ is plasma cyclotron frequency. The non-dimensional quantum diffraction parameter $H$ is defined as $H=\sqrt{\frac{\hbar^{2} \omega_{p i}^{2}}{m_{e, p} m_{i} C_{s i}^{4}}}$.

\section{III.THE LINEAR DAWS-DISPERSION RELATION}

To study the properties of linear DIA waves, Fourier transforming of the first order perturbed quantities of the Eqs. 1-7, we obtain the dispersion relation for DAWs in form of

$$
\omega^{4}-A \omega^{2}+B=0
$$


where $\omega$ and $k\left(k^{2}=k_{x}^{2}+k_{y}^{2}\right)$ are the normalized frequency and wave number, respectively, and

$$
\begin{aligned}
& A=\Omega^{2}\left(1-\mu^{2}\right)+k^{2} \sigma+\frac{k^{2}(1-\mu \beta d)}{S} \\
& B=\mu^{2} \Omega^{2}\left(\Omega^{2}+k^{2} \sigma\right)+\frac{\mu \beta d}{S}\left(\Omega^{2}+k^{4} \sigma\right) \\
& S=\frac{k^{2}+\mu_{d}(1-\beta d)+\left(1-\mu_{d}\right)(1-\beta d)}{\left(1+\frac{H^{2}}{4} k^{2}\right)}
\end{aligned}
$$

Note that, we assume the Fermi temperatures of electrons and positrons to be equal $(\sigma=\gamma)$. Fig. 1 shows the plot of dispersion relation for DIA waves.

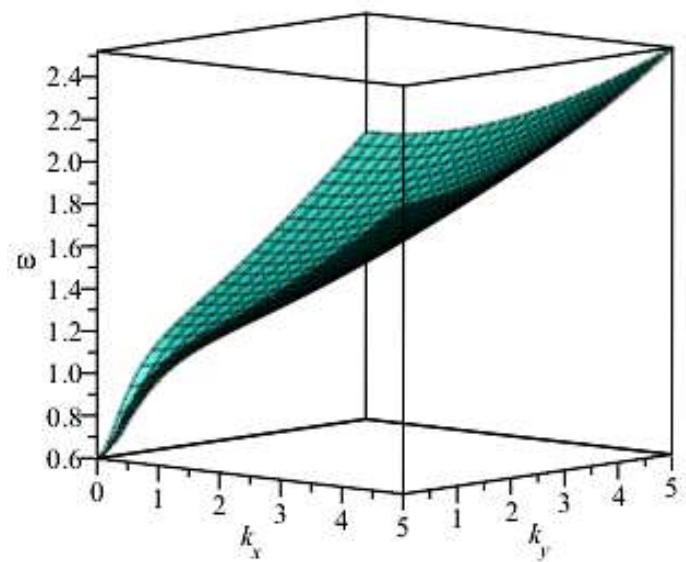

Fig. 1. Plot of dispersion relation of linear DIAWs with fixed values of $\Omega=0.6, \gamma=1, \alpha=20^{\circ}$, $\mu=3 \times 10^{-3}, u_{0}=1, \mu_{d}=0.5 H=2.11 \times 10^{-3}$,

\section{IV.THE NONLINEAR DAWS- LIMIT AND SMALL AMPLITUDE}

We employ the reductive perturbation method to Eqs. 1-7 to obtain the nonlinear ZakharovKuznetsov (ZK) equation for DIA waves. For This purpose, plasma parameters can be expanded in powers of $\varepsilon$ as

$$
\begin{aligned}
& u_{j z}=\varepsilon u_{j 1 z}+\varepsilon^{2} u_{j 2 z}+\ldots \\
& u_{j y}=\varepsilon^{\frac{3}{2}} u_{j 1 y}+\varepsilon^{2} u_{j 2 y}+\ldots \\
& u_{j x}=\varepsilon^{\frac{3}{2}} u_{j 1 x}+\varepsilon^{2} u_{j 2 x}+\ldots \\
& \varphi=\varepsilon \varphi_{1}+\varepsilon^{2} \varphi_{2}+\ldots
\end{aligned}
$$

where $\varepsilon$ is a small parameter measuring the weakness of the dispersion and $V_{0}$ is the wave phase speed normalized by the ion acoustic speed $\left(C_{s i}\right)$. We also introduce the following independent variables scaled as $X=\varepsilon^{\frac{1}{2}} x$, $Y=\varepsilon^{\frac{1}{2}} y, \quad Z=\varepsilon^{\frac{1}{2}}\left(z-V_{0} t\right) \quad$ and $\quad \tau=\varepsilon^{\frac{3}{2}} t$. Substituting Eq. 8 into Eqs. 1-7 and collecting the various order terms of $\varepsilon$, we obtain a ZK equation

$$
\begin{aligned}
& \frac{\partial \varphi_{1}}{\partial \tau}+A_{1} \varphi_{1} \frac{\partial \varphi_{1}}{\partial Z}+A_{2} \frac{\partial^{3} \varphi_{1}}{\partial Z^{3}}+ \\
& A_{3} \frac{\partial}{\partial Z}\left(\frac{\partial^{2} \varphi_{1}}{\partial X^{2}}+\frac{\partial^{2} \varphi_{1}}{\partial Y^{2}}\right)=0
\end{aligned}
$$

with the coefficients

$$
\begin{aligned}
A_{1}=\frac{1}{D} & -\mu_{d}(1-d)-\frac{\left(1-\mu_{d}\right)(1-d)}{\gamma^{2}}+\frac{2 \mu^{2} d}{V_{0}^{4}}- \\
& \left.-\frac{2 V_{0}^{2}+\sigma}{\left(V_{0}^{2}-\sigma\right)^{3}}\right] \\
A_{2}= & \frac{1}{D}\left(\frac{\mu_{d}(1-d) H^{2}}{4}-\frac{\left(1-\mu_{d}\right)(1-d) H^{2}}{4 \gamma^{2}}-1\right) \\
A_{3}= & \frac{1}{D}\left[\frac{\mu_{d}(1-d) H^{2}}{4}-\frac{\left(1-\mu_{d}\right)(1-d) H^{2}}{4 \gamma^{2}-\frac{d}{\mu \Omega^{2}}-}\right. \\
- & \left.\frac{V_{0}^{2}}{\left(V_{0}^{2}-\sigma\right)}\left(\frac{1}{\Omega^{2}}+\frac{\sigma}{\Omega^{2}\left(V_{0}^{2}-\sigma\right)}\right)-1\right]
\end{aligned}
$$

\section{Solitary Waves Solution OF THE ZK EQUATION}

To study the properties of the solitary waves propagating in a direction making an angle $\delta$ with the $Z$-axis, i.e., with the external magnetic field and lying in the $(Z-X)$ plane, we first rotate the coordinate axes $(X, Z)$ through an angle $\delta$, keeping the $\mathrm{Y}$ - axis fixed. Thus, we transform our independent variables to

$$
\begin{aligned}
& \zeta=X \cos \alpha-Z \sin \alpha \\
& \xi=X \sin \alpha+Z \cos \alpha \\
& \eta=Y
\end{aligned}
$$


$\frac{\partial}{\partial X}=\cos \alpha \frac{\partial}{\partial \zeta}+\sin \alpha \frac{\partial}{\partial \xi}$

$\frac{\partial}{\partial Y}=\frac{\partial}{\partial \eta}$

$\frac{\partial}{\partial Z}=\cos \alpha \frac{\partial}{\partial \xi}-\sin \alpha \frac{\partial}{\partial \zeta}$

This transformation of these independent variables allows us to write the $\mathrm{ZK}$ equation in the form

$$
\begin{aligned}
& \frac{\partial \varphi_{1}}{\partial \tau}+\alpha_{1} \varphi_{1} \frac{\partial \varphi_{1}}{\partial \xi}+\alpha_{2} \frac{\partial^{3} \varphi_{1}}{\partial \xi^{3}}+ \\
& \alpha_{3} \varphi_{1} \frac{\partial \varphi_{1}}{\partial \zeta}+\alpha_{4} \frac{\partial^{3} \varphi_{1}}{\partial \zeta^{3}}+\alpha_{5} \frac{\partial^{3} \varphi_{1}}{\partial \xi^{2} \partial \zeta}+\alpha_{6} \frac{\partial^{3} \varphi_{1}}{\partial \xi \partial \zeta^{2}} \\
& +\alpha_{7} \frac{\partial^{3} \varphi_{1}}{\partial \xi \partial \eta^{2}}+\alpha_{8} \frac{\partial^{3} \varphi_{1}}{\partial \zeta \partial \eta^{2}}=0
\end{aligned}
$$

We now look for a steady state solution of this $\mathrm{ZK}$ equation in the form $\varphi_{1}=\varphi_{0}(\eta)$ where $\eta=\xi-u_{0} \tau$ and $u_{0}$ is a constant speed normalized by the IA $\operatorname{speed}\left(C_{s i}\right)$.Using this transformation, Eq. 12 can be written as

$$
\frac{d \varphi_{0}}{d \tau}-u_{0} \frac{d \varphi_{0}}{d \eta}+\alpha_{1} \varphi_{0} \frac{d \varphi_{0}}{d \eta}+\alpha_{2} \frac{d^{3} \varphi_{0}}{d \eta^{3}}=0
$$

where

$$
\alpha_{1}=A_{1} \cos \alpha
$$

and

$\alpha_{2}=A_{2} \cos ^{3} \alpha+A_{3} \sin ^{2} \alpha \cos \alpha$. At stationary state $\frac{d \phi_{0}}{d \tau} \rightarrow 0$, so we can write the $\mathrm{ZK}$ equation in steady state form as

$$
-u_{0} \frac{d \varphi_{0}}{d \eta}+\alpha_{1} \varphi_{0} \frac{d \varphi_{0}}{d \eta}+\alpha_{2} \frac{d^{3} \varphi_{0}}{d \eta^{3}}=0
$$

Now, using the appropriate boundary conditions, viz., $\frac{d^{2} \varphi_{1}}{d \eta^{2}}, \frac{d \varphi_{1}}{d \eta}, \varphi_{1} \rightarrow 0$ at $|\eta| \rightarrow \infty$ one can write

$$
\frac{d \varphi_{0}}{d \eta}=\left(\sqrt{\frac{u_{0}}{\alpha_{2}}} \varphi_{0} \sqrt{1-\frac{\alpha_{1}}{3 u_{0}} \varphi_{0}}\right.
$$

Therefore, the solitary wave solution is given by

$$
\varphi_{0}(\eta)=\varphi_{m} \sec h^{2}(\kappa \eta)
$$

where $\varphi_{m}=\frac{3 u_{0}}{\alpha_{1}}$ is the amplitude and $\kappa=\sqrt{\frac{u_{0}}{4 \alpha_{2}}}$ is the inverse of the width $(\Delta)$ of the solitary waves. Figures 2-4 show the variation of the electrostatic potential $\varphi_{1}$ as a function of $\eta$. Figure 2 shows the effect of ion to electron Fermi temperature ratio $\sigma$ and $\mu_{d}$ on the amplitude of electrostatic potential $\varphi_{0}$.

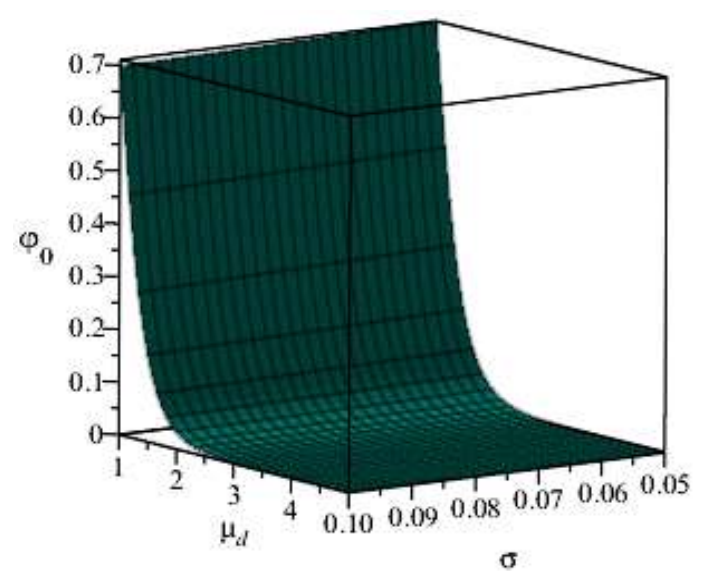

Fig. 2. Plot of electrostatic potential in terms of $\eta$ for different value of $\sigma$ and $\mu_{d}$ with fixed values $\gamma=1, \alpha=20^{\circ} \quad \mu=3 \times 10^{-3}, d=0.5$,
$H=2.11 \times 10^{-3}, u_{0}=1$

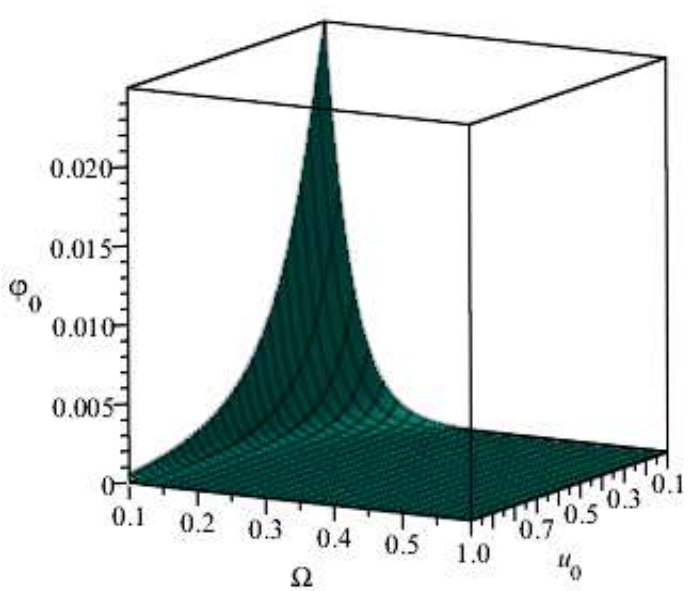

Fig. 3 Plot of electrostatic potential in terms of $\eta$ for different value of $\Omega$ and $u_{0}$ with fixed values $\sigma=0.1, \quad \gamma=1 \quad \alpha=20^{\circ}, \quad \Omega=0.6 \quad, \mu=3 \times 10^{-3}$, $\mu_{d}=5, d=0.5, H=2.11 \times 10^{-3}$ 
It is found that by increasing the density of dust grains $\left(\mu_{d}=1+\frac{p}{1-d}=\frac{e}{1-d}\right)$ and decreasing the fermi temperature of electrons $\left(\sigma=\frac{T_{F i}}{T_{F e}}\right)$, the amplitude of electrostatic potential decreases. Fig.3 indicates that increasing IA speed and decreasing plasma cyclotron frequency $\omega_{c i}\left(\Omega=\frac{\omega_{c i}}{\omega_{p i}}\right)$, lead to decreasing the amplitude of "electrostatic potential $\varphi_{0}$. In fig.4 we can observe DIA solitary wave and the effect of $\mu_{d}$.

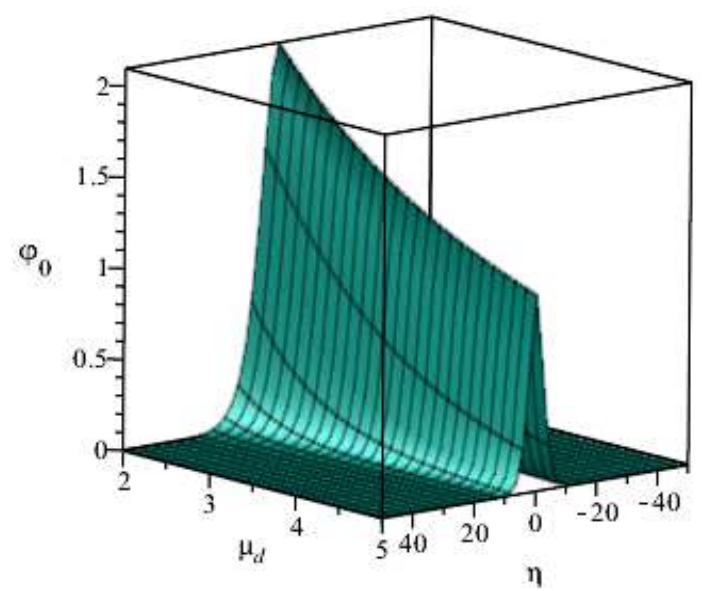

Fig. 4 Plot of electrostatic potential in terms of $\eta$ for different value of $\mu_{d}$ with fixed values $\Omega=0.6$ $\gamma=1, \quad \alpha=20^{\circ} \quad \mu=3 \times 10^{-3},, u_{0}=1, \quad \mu_{d}=5$, $H=2.11 \times 10^{-3}, d=0.5$

\section{CONCLUSION}

In this paper by using the quantum hydrodynamics model (QHD), the properties of nonlinear DIAWs are investigated in a magnetized quantum dusty plasma consists of inertialess electrons and positrons, cold ions and negatively charged dust grains. To study the small amplitude DIAWs, reductive perturbation method is applied and a quantum Zakharov-Kuznetsov(ZK) equation is derived. Solitary Waves Solution of the ZK Equation is presented. From numerical analysis, it is found that the amplitude of electrostatic potential decreases as $\sigma, \mu_{d}$ and $u_{0}$ increase. Additionally, the effect of $\Omega$ on this amplitude is shown. This work is aimed to study some basic features of DIAWs in dense magnetized quantum plasmas, which are ubiquitous in massive astrophysical objects.

\section{REFERENCES}

[1] R. Kodama, P. Norreys, K. Mima, A. Dangor, R. Evans, H. Fujita, Y. Kitagawa, K. Krushelnick, T. Miyakoshi, N. Miyanaga, T. Norimatsu, S. J. Rose, T. Shozaki, K. Shigemori, A. Sunahara, M. Tampo, K. A. Tanaka, Y. Toyama, T. Yamanaka, and M. Zepf, "Fast heating of ultrahigh-density plasma as a step towards laser fusion ignition," Nature, Vol. 412, pp. 798-802, 2001.

[2] M. Tabak, J. Hammer, M. E. Glinsky, W. L. Kruer, S. C. Wilks, J. Woodworth, E. M. Campbell, and M. D. Perry, "Ignition and high gain with ultrapowerful lasers," Phys. Plasmas, Vol. 1, pp. 1626-1634, 1994.

[3] H. Van Horn, "Dense astrophysical plasmas," Science, Vol. 252, pp. 384-389, 1991.

[4] L. Segretain, "Three-body crystallization diagrams and the cooling of white dwarfs," Astron. Astrophys. Vol. 310, pp. 485-488, 1996.

[5] M. Schlanges, M. Bonitz, and A. Tschttschjan, "Plasma Phase Transition in Fluid Hydrogen-Helium Mixtures," Contrib. Plasma Phys. Vol. 35, pp. 109-125, 1995.

[6] V. Filinov, M. Bonitz, W. Ebeling, and V. Fortov, "Thermodynamics of hot dense $\mathrm{H}$ plasmas: path integral Monte Carlo simulations and analytical approximations," Plasma Phys. Control. Fusion, Vol. 43, pp. 743-759, 2001.

[7] W. Ebeling and G. Norman, "Coulombic phase transitions in dense plasmas," J. Stat. Phys. Vol. 110, pp. 861-877, 2003.

[8] J. Vorberger, I. Tamblyn, S. A. Bonev, and B. Militzer, "Properties of dense fluid hydrogen and helium in giant gas planets," Contrib. Plasma Phys. Vol. 47, pp. 375-380, 2007.

[9] G. Chabrier, "Quantum effects in dense Coulumbic matter-Application to the cooling of white dwarfs," Astrophys. J. Vol. 414, pp. 695-700, 1993. 
[10] G. Manfredi and F. Haas, "Self-consistent fluid model for a quantum electron gas," Phys. Rev. B, Vol. 64, pp. 075316 (1-7), 2001.

[11]F. Haas, L. Garcia, J. Goedert, and G. Manfredi, "Quantum ion-acoustic waves," Phys. Plasmas, Vol. 10, pp. 3858-3866, 2003.

[12]A.-M. Wazwaz, "The extended tanh method for the Zakharov-Kuznetsov (ZK) equation, the modified ZK equation, and its generalized forms," Comm. Nonlinear Sci. Numer. Simulat. Vol. 13, pp. 1039-1047, 2008.

[13] A.-M. Wazwaz, "Exact solutions with solitons and periodic structures for the ZakharovKuznetsov (ZK) equation and its modified form," Commun. Nonlinear Sci. Numer. Simulat. Vol. 10, pp. 597-606, 2005.

[14] W. Moslem, S. Ali, P. K. Shukla, X. Tang, and G. Rowlands, "Solitary, explosive, and periodic solutions of the quantum ZakharovKuznetsov equation and its transverse instability," Phys. Plasmas, Vol. 14, pp. 082308 (1-5), 2007.

[15] S. Khan, A. Mushtaq, and W. Masood, "Dust ion-acoustic waves in magnetized quantum dusty plasmas with polarity effect," Phys. Plasmas, Vol. 15, pp. 013701 (1-5), 2008.

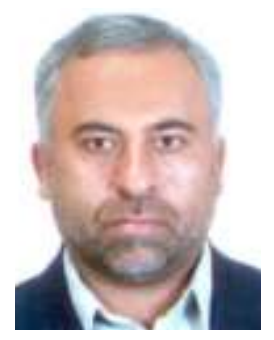

Hossein Zahed is an associate professor in plasma physics, from Sahand University of Technology, is graduated from University of Tabriz in plasma. His main research works is theoretical plasma physics. He is a member of Iran Nanotechnology, Physics Society of Iran and Optics and Photonics Society of Iran.

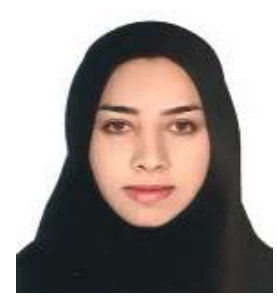

Elham Emadi received her M.Sc. degree in plasma physics from Sahand University of Technology in 2014. She is currently Ph.D. student at Sahand University of Technology. She is a member of Physics, Optics and Photonics Societies of Iran. 